\title{
Matching QCD and HQET at three loops
}

\author{
A.G. Grozin ${ }^{\mathrm{a}}$ \\ ${ }^{a}$ Institut für Theoretische Teilchenphysik, Karlsruher Institut für Technologie
}

QCD/HQET matching for the heavy-quark field [1] and heavy-light quark currents [2] with three-loop accuracy is discussed.

\section{Heavy-quark field}

QCD problems with a single heavy quark $Q$ can be treated in a simpler effective theory - HQET, if there exists a 4 -velocity $v$ such that the heavyquark momentum is $p=m v+k$ ( $m$ is the on-shell mass) and the characteristic residual momentum is small: $k \ll m$. QCD operators can be written as series in $1 / m$ via HQET operators; the coefficients in these series are determined by matching on-shell matrix elements in both theories.

At the tree level, the heavy-quark field $Q$ is related to the corresponding HQET field $Q_{v}$ (satisfying $\psi Q_{v}=Q_{v}$ ) by 34

$Q(x)=e^{-i m v \cdot x}\left(1+\frac{i \not D_{\perp}}{2 m}+\cdots\right) Q_{v}(x)$

$D_{\perp}^{\mu}=D^{\mu}-v^{\mu} v \cdot D$.

The matrix elements of the bare fields between the on-shell quark with momentum $p=m v+k$ and the vacuum in both theories are given by the on-shell wave-function renormalization constants:

$$
\begin{aligned}
& <0\left|Q_{0}\right| Q(p)>=\left(Z_{Q}^{\mathrm{os}}\right)^{1 / 2} u(p), \\
& <0\left|Q_{v 0}\right| Q(p)>=\left(\tilde{Z}_{Q}^{\mathrm{os}}\right)^{1 / 2} u_{v}(k)
\end{aligned}
$$

(HQET renormalization constants are denoted by $\tilde{Z})$. The Dirac spinors are related by the FoldyWouthuysen transformation

$u(m v+k)=\left[1+\frac{\not k}{2 m}+\mathcal{O}\left(\frac{k^{2}}{m^{2}}\right)\right] u_{v}(k)$.
Therefore, the bare fields are related by

$$
\begin{gathered}
Q_{0}(x)=e^{-i m v \cdot x}\left[z_{0}^{1 / 2}\left(1+\frac{i \not D_{\perp}}{2 m}\right) Q_{v 0}(x)\right. \\
\left.+\mathcal{O}\left(\frac{1}{m^{2}}\right)\right],
\end{gathered}
$$

where the bare matching coefficient is

$$
z_{0}=\frac{Z_{Q}^{\mathrm{os}}\left(g_{0}^{\left(n_{l}+1\right)}, a_{0}^{\left(n_{l}+1\right)}\right)}{\tilde{Z}_{Q}^{\mathrm{os}}\left(g_{0}^{\left(n_{l}\right)}, a_{0}^{\left(n_{l}\right)}\right)}
$$

(we use the covariant gauge: the gauge-fixing term in the Lagrangian is $-\left(\partial_{\mu} A_{0}^{a \mu}\right) /\left(2 a_{0}\right)$, and the free gluon propagator is $\left(-i / p^{2}\right)\left(g_{\mu \nu}-(1-\right.$ $\left.\left.a_{0}\right) p_{\mu} p_{\nu} / p^{2}\right)$; the number of flavours in $\mathrm{QCD}$ is $\left.n_{f}=n_{l}+1\right)$. The $\mathcal{O}(1 / m)$ matching coefficient in (3) is equal to the leading one, $z_{0}$; this reflexes the reparametrization invariance [5]. The $\overline{\mathrm{MS}}$ renormalized fields are related by the formula similar to (3), with the renormalized decoupling coefficient

$z(\mu)=\frac{\tilde{Z}_{Q}\left(\alpha_{s}^{\left(n_{l}\right)}(\mu), a^{\left(n_{l}\right)}(\mu)\right)}{Z_{Q}\left(\alpha_{s}^{\left(n_{l}+1\right)}(\mu), a^{\left(n_{l}+1\right)}(\mu)\right)} z_{0}$.

If there are no massive flavours except $Q$, then $\tilde{Z}_{Q}^{\text {os }}=1$ because all loop corrections are scale-free. The QCD on-shell renormalization constant $Z_{Q}^{\text {os }}$ contains the single scale $m$ in this case; it has been calculated 6 up to three loops. The three-loop $\overline{\mathrm{MS}}$ anomalous dimensions of $Q_{v}$ 67] and $Q$ [89] are also known. We have to express all three quantities $Z_{Q}^{\mathrm{os}}\left(g_{0}^{\left(n_{l}+1\right)}, a_{0}^{\left(n_{l}+1\right)}\right), Z_{Q}\left(\alpha_{s}^{\left(n_{l}+1\right)}(\mu), a^{\left(n_{l}+1\right)}(\mu)\right)$, $\tilde{Z}_{Q}\left(\alpha_{s}^{\left(n_{l}\right)}(\mu), a^{\left(n_{l}\right)}(\mu)\right)$ via the same variables, say, 
$\alpha_{s}^{\left(n_{l}\right)}(\mu), a^{\left(n_{l}\right)}(\mu)$, see 10. The explicit result for the renormalized matching coefficient $z(\mu)$ can be found in [1]. Gauge dependence first appears at three loops, as in $Z_{Q}^{\text {os }}$ [6]. The requirement of finiteness of the renormalized matching coefficient (5) at $\varepsilon \rightarrow 0$ has allowed the authors of [6] to extract $\tilde{Z}_{Q}$ from their result for $Z_{Q}^{\text {os }}$.

In the large- $\beta_{0}$ limit (see Chapter 8 in [11] for a pedagogical introduction):

$$
\begin{aligned}
z(\mu)= & +\int_{0}^{\beta} \frac{d \beta}{\beta}\left(\frac{\gamma(\beta)}{2 \beta}-\frac{\gamma_{0}}{2 \beta_{0}}\right) \\
& +\frac{1}{\beta_{0}} \int_{0}^{\infty} d u e^{-u / \beta} S(u)+\mathcal{O}\left(\frac{1}{\beta_{0}^{2}}\right),
\end{aligned}
$$

where $\beta=\beta_{0} \alpha_{s} /(4 \pi), \gamma=\gamma_{0} \alpha_{s} /(4 \pi)+\cdots$ (differences of $n_{l}$-flavour and $\left(n_{l}+1\right)$-flavour quantities can be neglected at the $1 / \beta_{0}$ order). The difference of the QCD and HQET anomalous dimensions $\gamma=\gamma_{Q}-\tilde{\gamma}_{Q}$ (it is gauge invariant at this order) and the Borel image $S(u)$ are [12]13[11]

$$
\begin{aligned}
& \gamma(\beta)=-2 \frac{\beta}{\beta_{0}} F(-\beta, 0)= \\
& 2 C_{F} \frac{\beta}{\beta_{0}} \frac{(1+\beta)\left(1+\frac{2}{3} \beta\right)}{B(2+\beta, 2+\beta) \Gamma(3+\beta) \Gamma(1-\beta)}, \\
& S(u)=\frac{F(0, u)-F(0,0)}{u}= \\
& -6 C_{F}\left[e^{(L+5 / 3) u} \frac{\Gamma(u) \Gamma(1-2 u)}{\Gamma(3-u)}\left(1-u^{2}\right)-\frac{1}{2 u}\right] .
\end{aligned}
$$

This Borel image has infrared renormalon poles at each positive half-integer $u$ and at $u=2$. Therefore, the integral in (6) is not well defined. Comparing its residue at the leading pole $u=1 / 2$ with the residue of the static-quark self-energy at its ultraviolet pole $u=1 / 2$ [14, we can express the renormalon ambiguity of $z(\mu)$ as

$\Delta z(\mu)=\frac{3}{2} \frac{\Delta \bar{\Lambda}}{m}$

( $\bar{\Lambda}$ is the ground-state meson residual energy). The matching coefficient is gauge invariant at the order $1 / \beta_{0}$. Expanding $\gamma(\beta)$ and $S(u)$ and integrating, we obtain confirm the contributions with the highest power of $n_{l}$ in each term in our threeloop result, and predict such a contribution at $\alpha_{s}^{4}$.
Numerically, in the Landau gauge at $n_{l}=4$

$$
\begin{aligned}
& z(m)=1-\frac{4}{3} \frac{\alpha_{s}^{(4)}(m)}{\pi} \\
& -(16.6629-4.5421)\left(\frac{\alpha_{s}^{(4)}(m)}{\pi}\right)^{2} \\
& -(153.4076+42.6271-61.5397)\left(\frac{\alpha_{s}^{(4)}(m)}{\pi}\right)^{3} \\
& -(1953.4013+\cdots)\left(\frac{\alpha_{s}^{(4)}(m)}{\pi}\right)^{4}+\cdots \\
& =1-\frac{4}{3} \frac{\alpha_{s}^{(4)}(m)}{\pi}-12.1208\left(\frac{\alpha_{s}^{(4)}(m)}{\pi}\right)^{2} \\
& -134.4950\left(\frac{\alpha_{s}^{(4)}(m)}{\pi}\right)^{3} \\
& -(1953.4013+\cdots)\left(\frac{\alpha_{s}^{(4)}(m)}{\pi}\right)^{4}+\cdots
\end{aligned}
$$

( $\beta_{0}$ is for $n_{l}=4$ flavours). Naive nonabelianization [12] works rather well at two and three loops. Numerical convergence of the series is very poor; this is related to the infrared renormalon at $u=1 / 2$.

Now let us consider the relation between the $\overline{\mathrm{MS}}$ renormalized electron field in QED and the Bloch-Nordsieck electron field. The bare matching coefficient $z_{0}=Z_{\psi}^{\text {os }}$ is gauge invariant to all orders, see 6 . In the Bloch-Nordsieck model, due to exponentiation, $\log \tilde{Z}_{\psi}=\left(3-a^{(0)}\right) \alpha^{(0)} /(4 \pi \varepsilon)$ (where the 0 -flavour $\alpha^{(0)}$ is equal to the onshell $\alpha \approx 1 / 137)$. In the full QED, $\log Z_{\psi}=$ $a^{(1)} \alpha^{(1)} /(4 \pi \varepsilon)+$ (gauge-invariant higher terms) (see [1] for the proof; this has been demonstrated up to four loops by the direct calculation [15]). The gauge dependence cancels in $\log \left(\tilde{Z}_{\psi} / Z_{\psi}\right)$ because of the QED decoupling relation $a^{(1)} \alpha^{(1)}=$ $a^{(0)} \alpha^{(0)}$. Therefore, the renormalized matching coefficient $z(\mu)$ in QED is gauge invariant to all orders. The three-loop result is presented in [1]. 


\section{Heavy-light currents}

Now we shall consider 2 MS renormalized heavy-light QCD quark currents

$j(\mu)=Z_{j}^{-1}(\mu) j_{0}, \quad j_{0}=\bar{q}_{0} \Gamma Q_{0}$,

where $\Gamma$ is a Dirac matrix. They can be expressed via operators in HQET

$$
\begin{aligned}
j(\mu)= & C_{\Gamma}(\mu) \tilde{\jmath}(\mu)+\frac{1}{2 m} \sum_{i} B_{i}(\mu) O_{i}(\mu) \\
& +\mathcal{O}\left(\frac{1}{m^{2}}\right)
\end{aligned}
$$

where

$\tilde{\jmath}(\mu)=\tilde{Z}_{j}^{-1}(\mu) \tilde{\jmath}_{0}, \quad \tilde{\jmath}_{0}=\bar{q}_{0} \Gamma Q_{v 0}$,

and $O_{i}$ are dimension-4 HQET operators with appropriate quantum numbers. The leading-order matching coefficients $C_{\Gamma}$ have been calculated up to two loops [12]16.

There are 8 Dirac structures giving nonvanishing quark currents in 4 dimensions:

$\Gamma=1, \quad \not, \quad \gamma_{\perp}^{\alpha}, \quad \gamma_{\perp}^{\alpha} \not$,

$\gamma_{\perp}^{[\alpha} \gamma_{\perp}^{\beta]}, \quad \gamma_{\perp}^{[\alpha} \gamma_{\perp}^{\beta]} \psi, \quad \gamma_{\perp}^{[\alpha} \gamma_{\perp}^{\beta} \gamma_{\perp}^{\gamma]}, \quad \gamma_{\perp}^{[\alpha} \gamma_{\perp}^{\beta} \gamma_{\perp}^{\gamma]} \psi$,

where $\gamma_{\perp}^{\alpha}=\gamma^{\alpha}-\not v^{\alpha}$. The last four of them can be obtained from the first four by multiplying by the 't Hooft-Veltman $\gamma_{5}^{\mathrm{HV}}$. We are concerned with flavour non-singlet currents only, therefore, we may also use the anticommuting $\gamma_{5}^{\mathrm{AC}}$ (there is no anomaly). The currents renormalized at a scale $\mu$ with different prescriptions for $\gamma_{5}$ are related by [17]

$$
\begin{aligned}
& \left(\bar{q} \gamma_{5}^{\mathrm{AC}} Q\right)_{\mu}=Z_{P}(\mu)\left(\bar{q} \gamma_{5}^{\mathrm{HV}} Q\right)_{\mu}, \\
& \left(\bar{q} \gamma_{5}^{\mathrm{AC}} \gamma^{\alpha} Q\right)_{\mu}=Z_{A}(\mu)\left(\bar{q} \gamma_{5}^{\mathrm{HV}} \gamma^{\alpha} Q\right)_{\mu}, \\
& \left(\bar{q} \gamma_{5}^{\mathrm{AC}} \gamma^{[\alpha} \gamma^{\beta]} Q\right)_{\mu}=Z_{T}(\mu)\left(\bar{q} \gamma_{5}^{\mathrm{HV}} \gamma^{[\alpha} \gamma^{\beta]} Q\right)_{\mu},
\end{aligned}
$$

where the finite renormalization constants $Z_{P, A, T}$ can be reconstructed from the differences of the anomalous dimensions of the currents. Multiplying $\Gamma$ by $\gamma_{5}^{\mathrm{AC}}$ does not change the anomalous dimension. In the case of $\Gamma=\gamma^{[\alpha} \gamma^{\beta]}$, multiplying it by $\gamma_{5}^{\mathrm{HV}}$ just permutes its components, and also does not change the anomalous dimension, therefore,

$Z_{T}(\mu)=1$

$Z_{P, A}(\mu)$ are known up to three loops [17.

The anomalous dimension of the HQET current (12) does not depend on the Dirac structure $\Gamma$. Therefore, there are no factors similar to $Z_{P, A}$ in HQET. Multiplying $\Gamma$ by $\gamma_{5}^{\mathrm{AC}}$ does not change the matching coefficient. Therefore, the matching coefficients for the currents in the second row of (13) can be obtained from those for the first row. In the $v$ rest frame

$$
\begin{aligned}
Z_{P}(\mu) & =\frac{C_{\gamma_{5}^{\mathrm{AC}}}(\mu)}{C_{\gamma_{5}^{\mathrm{HV}}}(\mu)}=\frac{C_{1}(\mu)}{C_{\gamma^{0} \gamma^{1} \gamma^{2} \gamma^{3}}(\mu)}, \\
Z_{A}(\mu) & =\frac{C_{\gamma_{5}^{\mathrm{AC}} \gamma^{0}}(\mu)}{C_{\gamma_{5}^{\mathrm{HV}} \gamma^{0}}(\mu)}=\frac{C_{\gamma^{0}}(\mu)}{C_{\gamma^{1} \gamma^{2} \gamma^{3}}(\mu)} \\
& =\frac{C_{\gamma_{5}^{\mathrm{AC}} \gamma^{3}}(\mu)}{C_{\gamma_{5}^{\mathrm{HV}} \gamma^{3}}(\mu)}=\frac{C_{\gamma^{3}}(\mu)}{C_{\gamma^{0} \gamma^{1} \gamma^{2}}(\mu)}, \\
Z_{T}(\mu) & =\frac{C_{\gamma_{5}^{\mathrm{AC}} \gamma^{0} \gamma^{1}}(\mu)}{C_{\gamma_{5}^{\mathrm{HV}} \gamma^{0} \gamma^{1}}(\mu)}=\frac{C_{\gamma^{0} \gamma^{1}}(\mu)}{C_{\gamma^{2} \gamma^{3}}(\mu)} \\
& =\frac{C_{\gamma_{5}^{\mathrm{AC}} \gamma^{2} \gamma^{3}}(\mu)}{C_{\gamma_{5}^{\mathrm{HV}} \gamma^{2} \gamma^{3}}(\mu)}=\frac{C_{\gamma^{2} \gamma^{3}}(\mu)}{C_{\gamma^{0} \gamma^{1}}(\mu)}=1 .
\end{aligned}
$$

In particular, $C_{\gamma_{\perp}}(\mu)=C_{\gamma_{\perp}^{[\alpha} \gamma_{\perp}^{\beta]}}(\mu)$. In the following we shall consider only the matching coefficients for the first 4 Dirac structures in (13).

In order to find the coefficients $C_{\Gamma}(\mu)$, we equate matrix elements of the left- and right-hand side of (11) from the heavy quark with momentum $p=m v+k$ to the light quark with momentum $k_{q}$ :

$$
\begin{aligned}
& <q\left(k_{q}\right)|j(\mu)| Q(m v+k)>= \\
& C_{\Gamma}(\mu)<q\left(k_{q}\right)|\tilde{\jmath}(\mu)| Q_{v}(k)>+\mathcal{O}\left(\frac{k, k_{q}}{m}\right) .
\end{aligned}
$$

The on-shell matrix elements are

$$
\begin{aligned}
<q\left(k_{q}\right)|j(\mu)| Q(p)>= & \bar{u}_{q}\left(k_{q}\right) \Gamma\left(p, k_{q}\right) u(p) \\
& \times Z_{j}^{-1}(\mu) Z_{Q}^{1 / 2} Z_{q}^{1 / 2} \\
<q\left(k_{q}\right)|\tilde{\jmath}(\mu)| Q_{v}(k)>= & \bar{u}_{q}\left(k_{q}\right) \tilde{\Gamma}\left(k, k_{q}\right) u_{v}(k) \\
& \times \tilde{Z}_{j}^{-1}(\mu) \tilde{Z}_{Q}^{1 / 2} \tilde{Z}_{q}^{1 / 2}
\end{aligned}
$$


where $\Gamma\left(p, k_{q}\right)$ and $\tilde{\Gamma}\left(k, k_{q}\right)$ are the bare vertex functions, and $\tilde{Z}_{q}$ differs from $Z_{q}$ because there are no $Q$ loops in HQET. The difference between $u(m v+k)$ and $u_{v}(k)$ is of order $k / m$, and can be neglected. It is most convenient to use $k=k_{q}=$ 0 , then the $\mathcal{O}(1 / m)$ term is absent. The QCD vertex has two Dirac structures:

$\Gamma(m v, 0)=\Gamma \cdot(A+B \psi)$.

This leads to

$\bar{u}(0) \Gamma(m v, 0) u(m v)=\bar{\Gamma}(m v, 0) \bar{u}(0) \Gamma u(m v)$,

$\bar{\Gamma}(m v, 0)=A+B$.

The HQET vertex has just one Dirac structure. Therefore,

$C_{\Gamma}(\mu)=\frac{\bar{\Gamma}(m v, 0) Z_{j}^{-1}(\mu) Z_{Q}^{1 / 2} Z_{q}^{1 / 2}}{\tilde{\Gamma}(0,0) \tilde{Z}_{j}^{-1}(\mu) \tilde{Z}_{Q}^{1 / 2} \tilde{Z}_{q}^{1 / 2}}$.

If all flavours except $Q$ are massless, all loop corrections to $\tilde{\Gamma}(0,0), \tilde{Z}_{Q}$, and $\tilde{Z}_{q}$ contain no scale and hence vanish: $\tilde{\Gamma}(0,0)=1, \tilde{Z}_{Q}=1$, $\tilde{Z}_{q}=1$. The quantities $\Gamma(m v, 0), Z_{Q}$, and $Z_{q}$ contain a single scale $m ; Z_{Q}$ has been calculated up to 3 loops in 6], $Z_{q}$ in [10, and $\Gamma(m v, 0)$ in the present work 2. The $\overline{\mathrm{MS}}$ renormalization constants $\tilde{Z}_{j}$ [7] and $Z_{j}$ [18. (for all $\Gamma$ ) are also known to 3 loops.

If there is another massive flavour ( $c$ in $b$-quark HQET), then $\tilde{\Gamma}(0,0), \tilde{Z}_{Q}$, and $\tilde{Z}_{q}$ contain a single scale $m_{c}$. The first two quantities have been calculated up to 3 loops in [19]; the last one is known from 10. The quantities $\Gamma(m v, 0), Z_{Q}$, and $Z_{q}$ now contain 2 scales, and are non-trivial functions of $x=m_{c} / m$. The renormalization constant $Z_{Q}$ has been calculated in this case, up to 3 loops, in 20 (the master integrals appearing in this case are discussed in Ref. 21]). The other two quantities are found in this work [2].

The bare on-shell QCD quantities $\bar{\Gamma}(m v, 0)$, $Z_{Q}$, and $Z_{q}$ are expressed via $g_{0}^{\left(n_{f}\right)}$ (and $m_{c 0}^{\left(n_{f}\right)}$ if it is non-zero; we re-express it via the onshell mass $m_{c}$ ). They don't contain $\mu$. The $\overline{\mathrm{MS}}$ QCD renormalization constant $Z_{j}$ is expressed via $\alpha_{s}^{\left(n_{f}\right)}(\mu)$. The bare on-shell HQET quantities $\tilde{\Gamma}(0,0), \tilde{Z}_{Q}$, and $\tilde{Z}_{q}$ are expressed via $g_{0}^{\left(n_{f}-1\right)}$ and $m_{c 0}^{\left(n_{f}-1\right)}$ (they are trivial at $m_{c}=0$ ); we reexpress $m_{c 0}^{\left(n_{f}-1\right)}$ via the on-shell mass $m_{c}$ (which is the same in both theories). These bare quantities also don't contain $\mu$. Finally, the $\overline{\mathrm{MS}}$ HQET renormalization constant $\tilde{Z}_{j}$ is expressed via $\alpha_{s}^{\left(n_{f}-1\right)}(\mu)$. We re-express all the quantities in (19) via $\alpha_{s}^{\left(n_{f}-1\right)}(\mu)$, see 10.

From equation of motion we have

$i \partial_{\alpha} j^{\alpha}=i \partial_{\alpha} j_{0}^{\alpha}=m_{0} j_{0}=m(\mu) j(\mu)$,

where $m(\mu)$ is the $\overline{\mathrm{MS}}$ mass of the heavy quark $Q$. Taking the on-shell matrix element between the heavy quark with $p=m v$ and the light quark with $k_{q}=0$ and re-expressing both QCD matrix elements via the matrix element of the HQET current with $\Gamma=1$, we obtain [12]

$m C_{\not}(\mu)=m(\mu) C_{1}(\mu)$.

The ratio $m(\mu) / m$ has been calculated at three loops numerically 22 and then analytically 23 . (the analytical results 236] were later independently confirmed in 24, and then in several other papers); for $m_{c} \neq 0, m(\mu) / m$ has been found in 20 .

The matching coefficients have been calculated up to 2 loops in [12, and to 3 loops in the present work 2. Analytical expressions are long; numerically, at $m_{c}=0$ and $\mu=m$ we have

$C_{1}^{(2)}=7.55+1.09=8.64$,

$C_{\prec}^{(2)}=-5.47+3.06=-2.41$,

$C_{\gamma_{\perp}}^{(2)}=-9.87+1.53=-8.34$,

$C_{\gamma_{\perp} \phi}^{(2)}=-14.13+2.42=-11.70$,

$C_{1}^{(3)}=64.74+75.34-38.16=101.92$,

$C_{\not}^{(3)}=-37.25-10.72+29.74=-18.23$,

$C_{\gamma_{\perp}}^{(3)}=-88.92-46.34+45.34=-89.92$,

$C_{\gamma_{\perp} \not}^{(3)}=-123.61-63.57+63.22=-123.96$

(in the middle part of each formula, terms with descending powers of $\beta_{0}^{\left(n_{f}-1\right)}$ are shown separately). Naive nonabelianization [12] works reasonably well. 
Table 1

Master integrals with 5 lines

\begin{tabular}{c|cccc} 
& & & & \\
& $5.1,5.1 \mathrm{a}$ & $5.2,5.2 \mathrm{a}$ & $5.3,5.3 \mathrm{a}$ & $5.4,5.4 \mathrm{a}$ \\
\hline$\varepsilon^{-3}$ & $\mathrm{DE}$ & $\mathrm{DE}$ & $\mathrm{DE}$ & $\mathrm{DE}$ \\
$\varepsilon^{-2}$ & $\mathrm{DE}$ & $\mathrm{DE}$ & $\mathrm{DE}$ & $\mathrm{DE}$ \\
$\varepsilon^{-1}$ & $\mathrm{DE}$ & $\mathrm{DE}$ & $\mathrm{DE}$ & $\mathrm{DE}$ \\
1 & $\mathrm{DE}$ & $\mathrm{NEW}$ & $\mathrm{MB}$ & $\mathrm{DE}$ \\
$\varepsilon$ & $\mathrm{DE}$ & $x$ & $x$ & $\mathrm{DE}$ \\
$\varepsilon^{2}$ & & & & $\mathrm{DE}$
\end{tabular}

At $m_{c} \neq 0$, results are expressed via the master integrals depending on $x=m_{c} / m$ [21. Their status is summarized in the Tables 1-4 in this paper. In the present work [2, we were able to obtain exact analytical expressions (via harmonic polylogarithms of $x$ ) for $\mathcal{O}(1)$ terms in the master integrals 5.2, 5.2a, from the requirement of finiteness of the matching coefficients. Therefore, the Table 3 in 21 should be now replaced with the following Table 1 (DE means the method of differential equations, and MB the Mellin-Barnes representation). Unfortunately, $\mathcal{O}(\varepsilon)$ terms in 4 master integrals are still known only as truncated series in $x$ (the entries $x$ in the table). Therefore, the $m_{c}$ corrections to the 3-loop matching coefficients are also known only as truncated series in $x$ (or numerical approximations).

We let's apply our results to the matrix elements between a $B$ or $B^{*}$ meson with momentum $p$ and the vacuum:

$<0\left|\left(\bar{q} \gamma_{5}^{\mathrm{AC}} Q\right)_{\mu}\right| B>=-i m_{B} f_{B}^{P}(\mu)$,

$<0\left|\bar{q} \gamma^{\alpha} \gamma_{5}^{\mathrm{AC}} Q\right| B>=i f_{B} p^{\alpha}$,

$<0\left|\bar{q} \gamma^{\alpha} Q\right| B^{*}>=i m_{B^{*}} f_{B^{*}} e^{\alpha}$,

$<0\left|\left(\bar{q} \sigma^{\alpha \beta} Q\right)_{\mu}\right| B^{*}>=f_{B^{*}}^{T}(\mu)\left(p^{\alpha} e^{\beta}-p^{\beta} e^{\alpha}\right)$.

The corresponding HQET matrix elements in the $v$ rest frame are

$<0\left|\left(\bar{q} \gamma_{5}^{\mathrm{AC}} Q_{v}\right)_{\mu}\right| B(\vec{k})>_{\mathrm{nr}}=-i F(\mu)$,

$<0\left|\left(\bar{q} \vec{\gamma} Q_{v}\right)_{\mu}\right| B^{*}(\vec{k})>_{\mathrm{nr}}=i F(\mu) \vec{e}$,

where the single-meson states are normalized by the non-relativistic condition

${ }_{\mathrm{nr}}<B\left(\vec{k}^{\prime}\right) \mid B(\vec{k})>_{\mathrm{nr}}=(2 \pi)^{3} \delta\left(\vec{k}^{\prime}-\vec{k}\right)$.
These two matrix elements are characterized by a single hadronic parameter $F(\mu)$ due to the heavyquark spin symmetry. From (20) we have 12 ]

$\frac{f_{B}^{P}(\mu)}{f_{B}}=\frac{m_{B}}{m(\mu)}$,

where we may replace $m_{B}$ by the on-shell $b$-quark mass $m$, neglecting power corrections.

Our main result is the ratio $f_{B^{*}} / f_{B}$. At $m_{c}=0$

$$
\begin{aligned}
& \frac{f_{B^{*}}}{f_{B}}=1-\frac{1}{2} C_{F} \frac{\alpha_{s}^{(4)}(m)}{\pi}+ \\
& \left(C_{F} r_{F}+C_{A} r_{A}+T_{F} n_{l} r_{l}+T_{F} r_{h}\right) C_{F}\left(\frac{\alpha_{s}^{(4)}(m)}{\pi}\right)^{2} \\
& +\left(C_{F}^{2} r_{F F}+C_{F} C_{A} r_{F A}+C_{A}^{2} r_{A A}+C_{F} T_{F} n_{l} r_{F l}\right. \\
& +C_{F} T_{F} r_{F h}+C_{A} T_{F} n_{l} r_{A l}+C_{A} T_{F} r_{A h} \\
& \left.+T_{F}^{2} n_{l}^{2} r_{l l}+T_{F}^{2} n_{l} r_{l h}+T_{F}^{2} r_{h h}\right) C_{F}\left(\frac{\alpha_{s}^{(4)}(m)}{\pi}\right)^{3} \\
& +\mathcal{O}\left(\alpha_{s}^{4}, \frac{\Lambda}{m}\right)
\end{aligned}
$$

where

$$
\begin{aligned}
& r_{F}=\frac{1}{3} \pi^{2} \log 2-\frac{1}{2} \zeta_{3}-\frac{4}{9} \pi^{2}+\frac{31}{48} \\
& r_{A}=-\frac{1}{6} \pi^{2} \log 2+\frac{1}{4} \zeta_{3}+\frac{1}{6} \pi^{2}-\frac{263}{144} \\
& r_{l}=\frac{19}{36}, \quad r_{h}=\frac{1}{9} \pi^{2}-\frac{41}{36}
\end{aligned}
$$




$$
\begin{aligned}
& r_{F F}=-\frac{8}{3} a_{4}-\frac{1}{9} \log ^{4} 2-\frac{2}{9} \pi^{2} \log ^{2} 2 \\
& +\frac{19}{6} \pi^{2} \log 2+\frac{25}{12} \zeta_{5}-\frac{1}{9} \pi^{2} \zeta_{3}+\frac{11}{8} \zeta_{3} \\
& -\frac{43}{1080} \pi^{4}-\frac{43}{24} \pi^{2}-\frac{289}{192} \text {, } \\
& r_{F A}=-\frac{20}{9} a_{4}-\frac{5}{24} \log ^{4} 2-\frac{5}{27} \pi^{2} \log ^{2} 2 \\
& +\frac{305}{108} \pi^{2} \log 2-\frac{115}{48} \zeta_{5}+\frac{1}{12} \pi^{2} \zeta_{3}-\frac{899}{144} \zeta_{3} \\
& +\frac{817}{12960} \pi^{4}-\frac{2233}{648} \pi^{2}+\frac{4681}{864} \text {, } \\
& r_{A A}=\frac{16}{9} a_{4}+\frac{2}{27} \log ^{4} 2+\frac{4}{27} \pi^{2} \log ^{2} 2 \\
& -\frac{119}{54} \pi^{2} \log 2+\frac{5}{6} \zeta_{5}-\frac{11}{144} \pi^{2} \zeta_{3}+\frac{343}{144} \zeta_{3} \\
& -\frac{17}{3240} \pi^{4}+\frac{2839}{1728} \pi^{2}-\frac{48125}{5184} \text {, } \\
& r_{F l}=\frac{16}{9} a_{4}+\frac{2}{27} \log ^{4} 2+\frac{4}{27} \pi^{2} \log ^{2} 2 \\
& -\frac{28}{27} \pi^{2} \log 2+\frac{25}{9} \zeta_{3}-\frac{11}{324} \pi^{4}+\frac{179}{162} \pi^{2}-\frac{815}{864}, \\
& r_{F h}=-\frac{32}{9} a_{4}-\frac{4}{27} \log ^{4} 2+\frac{4}{27} \pi^{2} \log ^{2} 2 \\
& +\frac{46}{27} \pi^{2} \log 2+5 \zeta_{3}-\frac{1}{162} \pi^{4}-\frac{1439}{1080} \pi^{2}-\frac{119}{36}, \\
& r_{A l}=\frac{8}{9} a_{4}-\frac{1}{27} \log ^{4} 2-\frac{2}{27} \pi^{2} \log ^{2} 2 \\
& +\frac{14}{27} \pi^{2} \log 2-\frac{13}{18} \zeta_{3}+\frac{13}{3240} \pi^{4}-\frac{17}{72}+\frac{422}{81}, \\
& r_{A h}=\frac{16}{9} a_{4}+\frac{2}{27} \log ^{4} 2-\frac{2}{27} \pi^{2} \log ^{2} 2 \\
& -\frac{86}{27} \pi^{2} \log 2+\frac{55}{48} \zeta_{5}-\frac{31}{144} \pi^{2} \zeta_{3}+\frac{43}{36} \zeta_{3} \\
& +\frac{8}{405} \pi^{4}+\frac{577}{270} \pi^{2}-\frac{1121}{648} \\
& r_{l l}=-\frac{1}{27} \pi^{2}-\frac{203}{324}, \quad r_{l h}=\frac{5}{81} \pi^{2}-\frac{101}{162}, \\
& r_{h h}=-\frac{8}{9} \zeta_{3}+\frac{8}{405} \pi^{2}+\frac{277}{324}
\end{aligned}
$$

$\left(a_{4}=\operatorname{Li}_{4}(1 / 2)\right)$. The result for $f_{B^{*}}^{T}(m) / f_{B^{*}}$ is similar.
Numerically,

$$
\begin{aligned}
& \left(\frac{f_{B^{*}}}{f_{B}}\right)^{(2)}=-4.40-1.97=-6.37, \\
& \left(\frac{f_{B^{*}}^{T}\left(m_{b}\right)}{f_{B^{*}}}\right)^{(2)}=-4.26+0.89=-3.37, \\
& \left(\frac{f_{B^{*}}}{f_{B}}\right)^{(3)}=-51.67-42.21+16.33=-77.55, \\
& \left(\frac{f_{B^{*}}^{T}\left(m_{b}\right)}{f_{B^{*}}}\right)^{(3)}=-34.69-22.91+19.07 \\
& =-38.53 .
\end{aligned}
$$

Naive nonabelianization [12] works reasonably well.

Asymptotics of the perturbative coefficients for the matching coefficients at a large number of loops $l \gg 1$ have been investigated in Ref. [25] in a model-independent way. The results contain three unknown normalization constants $N_{0,1,2} \sim$ 1. The asymptotics of the perturbative coefficients for $f_{B^{*}} / f_{B}$ contain $N_{0}$ and $N_{2}$; in the case of $m / \hat{m}$ it contains only $N_{0}$ :

$$
\begin{aligned}
& \left(\frac{f_{B^{*}}}{f_{B}}\right)_{L=-5 / 3}^{(n+1)}=-\frac{14}{27}\left\{1+\mathcal{O}\left(\frac{1}{n}\right)\right. \\
& \left.+\frac{2}{7}\left(\frac{50}{3} n\right)^{-9 / 25}\left[1+\mathcal{O}\left(\frac{1}{n}\right)\right] \frac{N_{2}}{N_{0}}\right\} \\
& \times\left(\frac{m}{\hat{m}}\right)_{L=-5 / 3}^{(n+1)} .
\end{aligned}
$$

The coefficient of $N_{2} / N_{0}$ is about 0.08 at $n=2$, and it seems reasonable to neglect this contribution. Neglecting also $1 / n$ corrections, we obtain 25$]$

$$
\left(\frac{f_{B^{*}}}{f_{B}}\right)_{L=-5 / 3}^{(3)}=-\frac{14}{27} \cdot 56.37=-29.23 .
$$

Our exact result -37.787 agrees with this prediction reasonably well. However, $1 / n$ corrections are large and tend to break this agreement. It is natural to expect that $1 / n^{2}$ (and higher) corrections are also substantial at $n=2$.

Acknowledgements. I am grateful to S. Bekavac, P. Marquard, J.H. Piclum, D. Seidel, 
M. Steinhauser for collaboration on 2, and to D.J. Broadhurst, K.G. Chetyrkin, D.V. Shirkov, V.A. Smirnov for useful discussions.

\section{REFERENCES}

1. A.G. Grozin, arXiv:1004.2662 [hep-ph].

2. S. Bekavac, A.G. Grozin, P. Marquard, J.H. Piclum, D. Seidel, M. Steinhauser, Nucl. Phys. B 833 (2010) 46 arXiv:0911.3356 [hep$\mathrm{ph}]$.

3. C.L.Y. Lee, Preprint CALT-68-1663, Caltech (1991).

4. J.G. Körner, G. Thompson, Phys. Lett. B 264 (1991) 185.

5. M.E. Luke, A.V. Manohar, Phys. Lett. B 286 (1992) 348.

6. K. Melnikov, T. van Ritbergen, Nucl. Phys. B 591 (2000) 515 arXiv:hep-ph/0005131.

7. K.G. Chetyrkin, A.G. Grozin, Nucl. Phys. B 666 (2003) 289 arXiv:hep-ph/0303113.

8. O.V. Tarasov, Preprint JINR P2-82-900, Dubna (1982).

9. S.A. Larin, J.A.M. Vermaseren, Phys. Lett. B 303 (1993) 334 arXiv:hep-ph/9302208.

10. K.G. Chetyrkin, B.A. Kniehl, M. Steinhauser, Nucl. Phys. B 510 (1998) 61 arXiv:hep-ph/9708255.

11. A.G. Grozin, Heavy Quark Effective Theory, Springer Tracts in Modern Physics 201, Springer (2004).

12. D.J. Broadhurst, A.G. Grozin, Phys. Rev. D 52 (1995) 4082 arXiv:hep-ph/9410240].

13. M. Neubert, C.T. Sachrajda, Nucl. Phys. B 438 (1995) 235 arXiv:hep-ph/9407394.

14. M. Beneke, V.M. Braun, Nucl. Phys. B 426 (1994) 301 arXiv:hep-ph/9402364.

15. K.G. Chetyrkin, A. Rétey, Nucl. Phys. B $\mathbf{5 8 3}$ (2000) 3 arXiv:hep-ph/9910332.

16. A.G. Grozin, Phys. Lett. B 445 (1998) 165 arXiv:hep-ph/9810358.

17. S.A. Larin, J.A.M. Vermaseren, Phys. Lett. B 259 (1991) 345; S.A. Larin, in Quarks92, ed. D.Yu. Grigoriev, V.A. Matveev, V.A. Rubakov, P.G. Tinyakov, World Scientific (2003) p. 201 arXiv:hep-ph/9302240.

18. J.A. Gracey, Phys. Lett. B 488 (2000) 175 arXiv:hep-ph/0007171.
19. A.G. Grozin, A.V. Smirnov, V.A. Smirnov, JHEP 11 (2006) 022 arXiv:hep-ph/0609280.

20. S. Bekavac, A.G. Grozin, D. Seidel, M. Steinhauser, JHEP 10 (2007) 006 arXiv:0708.1729 [hep-ph]].

21. S. Bekavac, A.G. Grozin, D. Seidel, V.A. Smirnov, Nucl. Phys. B 819 (2009) 183 arXiv:0903.4760 [hep-ph]].

22. K.G. Chetyrkin, M. Steinhauser, Phys. Rev. Lett. 83 (1999) 4001 arXiv:hep-ph/9907509; Nucl. Phys. B 573 (2000) 617 arXiv:hep-ph/9911434.

23. K. Melnikov, T. van Ritbergen, Phys. Lett. B 482 (2000) 99 arXiv:hep-ph/9912391.

24. P. Marquard, L. Mihaila, J.H. Piclum, M. Steinhauser, Nucl. Phys. B 773 (2007) 1 arXiv:hep-ph/0702185.

25. F. Campanario, A.G. Grozin, T. Mannel, Nucl. Phys. B 663 (2003) 280; Erratum: ibid. B 670 (2003) 331 arXiv:hep-ph/0303052. 